\title{
Geldanamycin induces CHOP expression through a 4-(2-aminoethyl)-benzenesulfonyl fluoride-responsive serine protease
}

\author{
Toru Hosoi ${ }^{1, *}$, Kanae Hyoda ${ }^{2, *}$, Yasunobu Okuma ${ }^{3}$, Yasuyuki Nomura ${ }^{2,4}$, Koichiro Ozawa ${ }^{1}$ \\ ${ }^{1}$ Department of Pharmacotherapy, Graduate School of Biomedical Sciences, Hiroshima University, 1-2-3 Kasumi, Minami-ku, Hiro- \\ shima 734-8551, Japan; ${ }^{2}$ Department of Pharmacology, Graduate School of Pharmaceutical Sciences, Hokkaido University, Sapporo \\ 060-0812, Japan; ${ }^{3}$ Department of Pharmacology, Faculty of Pharmaceutical Sciences, Chiba Institute of Sciences, Choshi, Chiba \\ 288-0025, Japan; ${ }^{4}$ Yokohama College of Pharmacy, Yokohama, Kanagawa 245-0066, Japan
}

Cell Research (2007) 17: 184-186. doi: 10.1038/sj.cr.7310122; published online 23 January 2007

\section{Dear Editor:}

Geldanamycin is a benzoquinone ansamycin, which was originally described as a tyrosine kinase inhibitor. However, subsequent studies have revealed that geldanamycin binds to and inhibits heat-shock protein 90 (Hsp90) activity [1]. Hsp90 is a molecular chaperone involved in the conformational maturation of proteins such as mutated $\mathrm{p} 53$, Raf-1, Akt, Bcr-Abl, and ErbB2. It is suggested that agents inhibiting Hsp90 have anti-cancer properties, although the precise molecular mechanisms underlying the anti-cancer effects of geldanamycin are not well understood.

Increasing evidence has suggested that diabetes and neurodegenerative disorders such as Parkinson's and Alzheimer's diseases are related to the disruption of endoplasmic reticulum (ER) function. In response to ER stress, unfolded proteins accumulate and aggregate in the $\mathrm{ER}$, which will trigger many rescuer responses, including the unfolded protein response (UPR) and ER-associated degradation. Interestingly, geldanamycin has been shown to upregulate ER chaperones and the expression of $\mathrm{CHOP}$ [2]. Moreover, Hsp90 associates with PERK and IRE1 $\alpha$, ER-resident trans-membrane protein kinases involved in ER stress response [3]. These observations suggest that Hsp90 is involved in ER stress response and may play an important role in ER stress-related diseases. Therefore,

*These two authors contributed equally to this work

Correspondence: Koichiro Ozawa

Tel/Fax: +81-82-257-5332;

E-mail: ozawak@hiroshima-u.ac.jp we investigated the potential mechanism by which geldanamycin affects ER stress using the L929 fibroblastoma cell line.

The UPR is characterized by activation of the signal transduction pathway triggered by IRE1. Activation of IRE1 induces X-box binding protein (XBP-1) mRNA splicing [4]. XBP-1 protein produced from the spliced mRNA then functions as a transcription factor to induce ER-stress genes [4]. The XBP-1 splicing was observed in geldanamycin-treated cells in our experiments, indicating that geldanamycin induces the ER stress response pathway (data not shown). We further investigated whether geldanamycin induces CHOP expression in L929 cells. As assessed by RT-PCR (see Supplement 1 for Materials and Methods), we observed an increase in the CHOP transcript in geldanamycin-treated cells $(10 \mu \mathrm{M}, 2 \mathrm{~h}$ ) (Figure 1D and $1 \mathrm{E})$. The CHOP protein level was also significantly induced at $6 \mathrm{~h}$ after geldanamycin treatment (Figure $1 \mathrm{~F}$ and $1 \mathrm{G}$ ).

We next investigated intracellular mechanisms of geldanamycin-induced CHOP expression (see Supplement 1 for Materials and Methods). The PERK-eIF2 $\alpha$ pathway has been reported to be involved in ER stress-induced CHOP expression [5]. Thus, we investigated whether geldanamycin can induce eIF $2 \alpha$ phosphorylation. As a positive control, the ER stress-inducing reagent $\operatorname{Tg}(3 \mu \mathrm{M}$, $2 \mathrm{~h}$ ) caused an increase in eIF $2 \alpha$ phosphorylation in L929 cells (Figure 1A). However, geldanamycin did not increase eIF2 $\alpha$ phosphorylation at all examined time points (Figure $1 \mathrm{~B}$ and 1C). Geldanamycin suppresses the binding of 3phosphoinositide-dependent protein kinase-1 (PDK1) to Hsp90 and inhibits the activation of Akt [6]. As shown in Figure 1B, Akt phosphorylation was inhibited in geldana- 
A

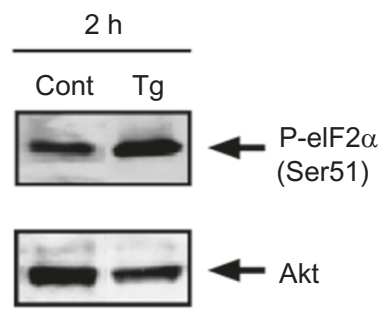

B
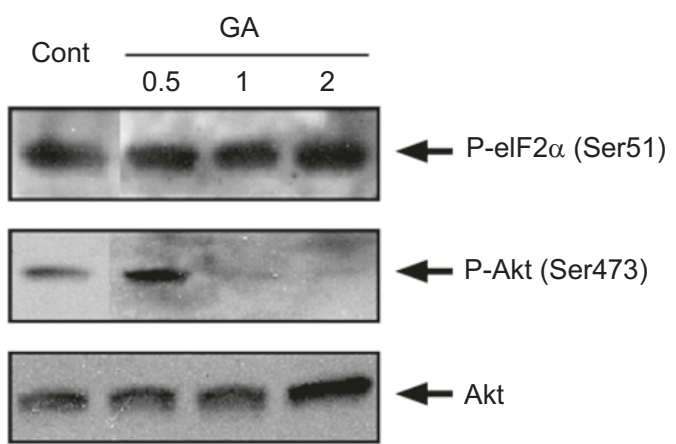

C

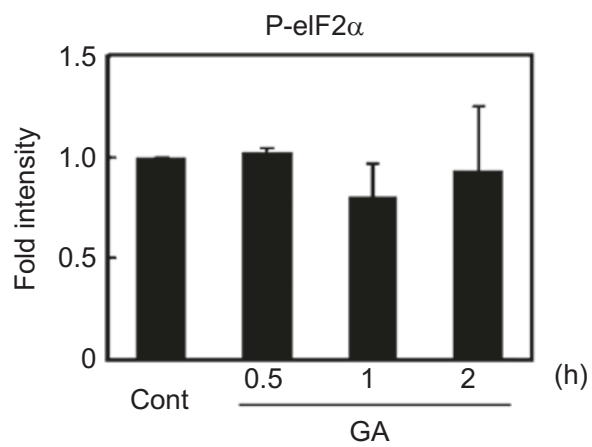

$\mathrm{D}$

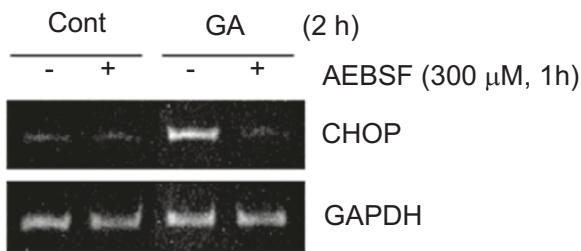

$E$

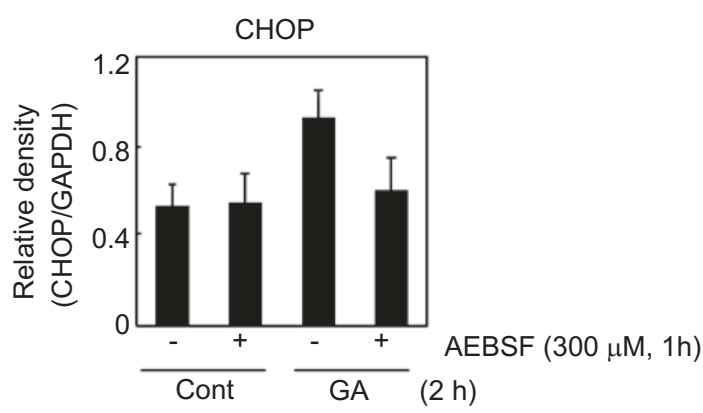

$\mathrm{F}$

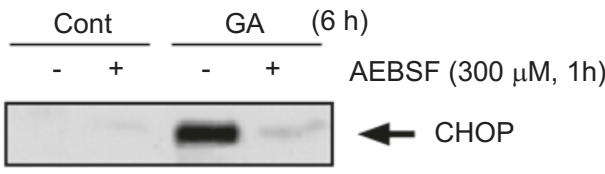

G

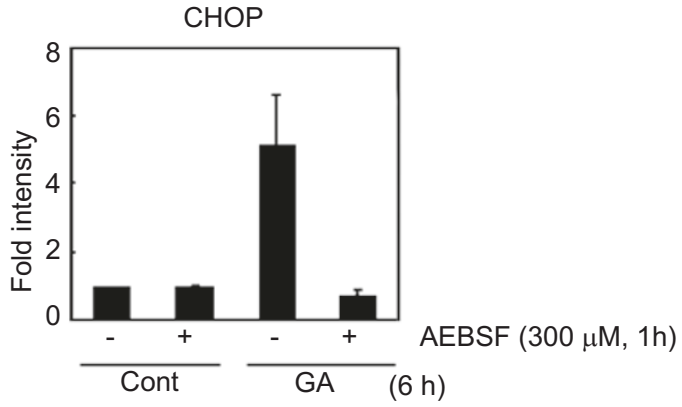

Figure 1 (A) L929 cells were treated with thapsigargin (Tg: $3 \mu \mathrm{M})$ for $2 \mathrm{~h}$ and Western blotting was performed using antibodies against phospho-eIF2 $\alpha$ (Ser51) and Akt. (B) Cells were treated with geldanamycin $(\mathrm{GA}, 10 \mu \mathrm{M})$ for the indicated time periods. Phospho-eIF2 $\alpha$ (Ser51), phospho-Akt (Ser473), and Akt were detected by Western blotting. (C) The expression levels are expressed as the fold intensity compared with the control group. (D) Cells were pretreated with AEBSF (300 $\mu \mathrm{M})$ for $1 \mathrm{~h}$ and then stimulated with geldanamycin $(\mathrm{GA}, 10 \mu \mathrm{M})$ for $2 \mathrm{~h}$. RT-PCR was performed using specific primers for each mRNAs. (E) The levels of CHOP mRNA were quantified by densitometric analysis and normalized to that of the corresponding GAPDH internal control. (F) Cells were pretreated with AEBSF $(300 \mu \mathrm{M})$ for $1 \mathrm{~h}$ and then stimulated with geldanamycin $(\mathrm{GA}, 10 \mu \mathrm{M})$ for $6 \mathrm{~h}$. CHOP protein induction was detected by Western blotting. (G) The levels of CHOP protein were quantified by densitometric analysis and are presented as the fold intensity compared with the control group. 
mycin-treated cells, indicating that geldanamycin functions effectively under our experimental conditions. These results suggest that eIF $2 \alpha$ is not involved in geldanamycin-induced CHOP expression.

Serine protease has been suggested to be involved in ER stress-related diseases [7, 8]. Thus, we investigated the effect of 4-(2-aminoethyl)-benzenesulfonyl fluoride (AEBSF) on geldanamycin-induced CHOP expression. AEBSF is a potent serine protease inhibitor, which acts by sulfonylation of the serine residue at the active site [9]. As shown in Figure 1D and 1E, geldanamycin-induced CHOP transcription was completely inhibited by AEBSF. The inhibitory effect of AEBSF on geldanamycin-induced CHOP expression was also confirmed at the protein level (Figure $1 \mathrm{~F}$ and $1 \mathrm{G})$. These results suggest that a serine protease is involved in geldanamycin-induced CHOP expression. ER stress has been shown to lead to the activation of activating transcription factor 6 (ATF6), and the process involves the cleavage of ATF 6 by a serine protease called site- 1 protease (S1P) [8]. The cytoplasmic domain of ATF6 has been reported to induce CHOP expression [10]. Moreover, AEBSF has been reported to inhibit the activation of ATF6 through inhibiting S1P [7]. Thus, it seems likely that the inhibitory effect of AEBSF on geldanamycin-induced CHOP expression is mediated by inhibition of ATF6 activation.

The present results raise the possibility that one of the mechanisms of the anti-cancer effect of geldanamycin may be mediated through CHOP induction. Our results also suggest that AEBSF is a unique and interesting compound with respect to ER stress response, and its detailed pharmacological role awaits to be investigated in the future. Nevertheless, the present study provides new insight into the molecular mechanism of geldanamycin-induced cell dysfunction, which could be important for understanding cancer and/or ER stress-related diseases.

\section{Acknowledgments}

This research was supported by Grants-in-Aid for Sci- entific Research from the Ministry of Education, Science, Sports, and Culture, Japan, and by the Mochida Memorial Foundation for Medical and Pharmaceutical Research.

\section{References}

1 Whitesell L, Mimnaugh EG, De Costa B, Myers CE, Neckers LM. Inhibition of heat shock protein HSP90-pp60v-src heteroprotein complex formation by benzoquinone ansamycins: essential role for stress proteins in oncogenic transformation. Proc Natl Acad Sci USA 1994; 91:8324-8328.

2 Lawson B, Brewer JW, Hendershot LM. Geldanamycin, an hsp90/GRP94-binding drug, induces increased transcription of endoplasmic reticulum (ER) chaperones via the ER stress pathway. J Cell Physiol 1998; 174:170-178.

3 Marcu MG, Doyle M, Bertolotti A, Ron D, Hendershot L, Neckers L. Heat shock protein 90 modulates the unfolded protein response by stabilizing IRE1a. Mol Cell Biol 2002; 22:85068513.

4 Yoshida H, Matsui T, Yamamoto A, Okada T, Mori K. XBP1 mRNA is induced by ATF6 and spliced by IRE1 in response to ER stress to produce a highly active transcription factor. Cell 2001; 107:881-891.

5 Harding HP, Novoa I, Zhang Y, et al. Regulated translation initiation controls stress-induced gene expression in mammalian cells. Mol Cell 2000; 6:1099-1108.

6 Fujita N, Sato S, Ishida A, Tsuruo T. Involvement of Hsp90 in signaling and stability of 3-phosphoinositide-dependent kinase-1. J Biol Chem 2002; 277:10346-10353.

7 Okada T, Haze K, Nadanaka S, et al. A serine protease inhibitor prevents endoplasmic reticulum stress-induced cleavage but not transport of the membrane-bound transcription factor ATF6. J Biol Chem 2003; 278:31024-31032.

8 Ye J, Rawson RB, Komuro R, et al. ER stress induces cleavage of membrane-bound ATF6 by the same proteases that process SREBPs. Mol Cell 2000; 6:1355-1364.

9 Markwardt F, Drawert J, Walsmann P. Synthetic low molecular weight inhibitors of serum kallikrein. Biochem Pharmacol 1974; 23: $2247-2256$.

10 Yoshida H, Okada T, Haze K, et al. ATF6 activated by proteolysis binds in the presence of NF-Y (CBF) directly to the cis-acting element responsible for the mammalian unfolded protein response. Mol Cell Biol 2000; 20:6755-6767.

(Supplementary Information is linked to the online version of the paper on the Cell Research website.) 